\title{
Monitoring of Misalignment in Low Speed Geared Shafts with Acoustic Emission Sensors
}

\author{
Enrique Caso* A Alfonso Fernandez-del-Rincon - Pablo Garcia • \\ Miguel Iglesias • Fernando Viadero
}

Received: date / Accepted: date

\begin{abstract}
The most common condition monitoring method applied to rotating machines is vibration measurement, although recently it has been proposed that acoustic emission $(A E)$ offers additional advantages in early fault detection and low speed rotation systems. Capturing the $A E$ signals is conditioned by the severe attenuation with distance and the presence of material discontinuities between source and sensor, therefore measurement device placement is more important than in traditional accelerometry. In this study $A E$ signals are measured in gearboxes at misalignment conditions for the revision of signal characteristics from the generated signal due to the interaction of shafts, bearings and gears. For this purpose, several tests are performed using combinations of speed and varying load, considering the effect of lubricating oil temperature. Fixed measurements are taken over the bench supports, and on-board, where the sensors are rotating with the shafts. In parallel to acoustic emission a vibration analysis is done in order to contrast and analyze differences between both technologies at different operation states. Acquired data is processed to obtain statistical parameters from measurements in order to verify the values and tendencies due to transient phenomena related to changes in speed and load. $A E$ provides better results than vibration employing the same time domain condition indicators. On the contrary, vibration recognizes clearly the fault through frequency analysis.
\end{abstract}

\footnotetext{
* Enrique Caso

Department of Structural and Mechanical Engineering. ETSIIT University of Cantabria

Avda. de los Castros s/n. 39005 Santander. Spain

E-mail: casoe@unican.es
}

Keywords acoustic emission · low speed · misalignment · gear transmissions · varying load

\section{Introduction}

The implementation of acoustic emission $(A E)$ in the field of condition maintenance of rotating machines has a long way to achieve the same status than the analogous vibration analysis. This technology was initially developed for static structures, as pressure gas tanks or bridge structures, and recently it has been imported to the monitoring of mechanisms with moving elements as bearings, gearboxes, pumps or engines. The initial use of this technique in static components allows the detection of the progression of small defects and its localization (via triangulation or advanced methods) $[1,2]$. In machines, defects are not the only source of emissions: the interacting components generate $A E$ in common operation. In this case, the application of $A E$ is not trivial due to the greater difficulties in the interpretation and processing of the acquired information [3]. Successful studies have been carried out in order to apply the $A E$ in the monitoring of gearboxes and bearings with promising advantages [4].

This technology is based in the high frequency elastic waves recorded by a piezoelectric sensor generated due to instant energy releases produced by strain, defects or the interaction between components in relative movement [5]. The waves propagates from the source in all directions across the material, suffering attenuation, reflection and dis- 
continuities. These perturbations reach the sensor on the surface of the material as Rayleigh waves, generating the signals when the piezoelectric element is affected with his displacement $[3,6]$. Randomness and noise presence on $A E$ signal, combined with gear transmission complexity, makes difficult the identification of failures. The interaction of each component produces an $A E$ source behaving like simultaneous independent emissions. The different components and subcomponents and the way they are fixed turn out to be interfaces between source and sensor.

$A E$ physical mechanisms related with the generation of the mechanic waves are not completely clear, being the asperity contact the main hypothesis $[7,8]$. That contact involving surface asperities has been noticed as the source of acoustic emissions in faulty bearings, showing an identifiable response before vibration is able to detect it, specially at very low rotational speeds [9-11]. Regarding to gear monitoring, the interaction and impact between meshing teeth are postulated as the generation origin of the elastic waves recorded as $A E$ signals due to the material deformation and consecutive energy release [12].

Previous studies have proposed the advantages of $A E$ versus traditional accelerometry at low speed due to the less amount of energy liberated by the defects [13]. Frequency of acoustic waves depend more on the material characteristics than on rotation speed [14]. The monitoring of low speed transmission components, as shafts, bearings and gearboxes, has a special interest because of the limitations of vibration analysis, in specially working with wind mills: its low speed stage operates in a range of $10 \mathrm{rpm}$ and varying loads because of the randomness effect of wind. A diminution of transmission failure rates by the employment of different condition monitoring systems is a trend in the wind energy industry [15]. Misalignment is a common cause of machine vibration being cause of $30 \%$ machines down time [16]. Wind turbine shaft alignment is necessary for a reliable and optimal long term operation [17]. Defects in components, as bearings or gears, of the wind turbine transmission could be originated by misalignment [18].

$A E$ signals are acquired at very high sample rates, over $1 \mathrm{MHz}$, it produces big files with a huge amount of data. Specially working at low speed, considering that a complete revolution lasts several seconds. At low speed rates, the frequency analysis is more complex, due the required spectral resolution for the detection and diagnosis, when the mechanical frequencies are closer, even talking about vibrations [19]. Computational methods or increasing the acquisition time are a solution when limitations are not present [20]. The downside of the low speed has driven specific methods to improve the diagnosis with $A E$ in bearing monitoring [21, 22 ] or the data amount reduction [23]. New signals are created through the raw emissions employing filters, envelopes and other algorithms in order to extract further information, as low frequency content or the reduction of signal size. This processed signals are useful when $A E$ signatures shows burst, exceeding clearly the noise threshold at periodic intervals, as has been seen in planetary gears but not in the studied transmission, where the signals has very low amplitude.

A main factor in $A E$ monitoring is the sensor location: signal is attenuated by interfaces and is affected by the transmission properties of the materials it is going through. The recorded information by the $A E$ sensor varies greatly if it has been placed in direct contact to the gear surface or over a bearing casing [24]. Placing a sensor in a moving element inside a complex system is not always a possibility, being forced to install on the outside of the machinery, but in some investigations of bearings and gears defects response is showed from the casings [25].

This research is linked with the development of a low cost condition monitoring device for offshore windmills employing acoustic emission (PredictEA project). High speed transmission components and generator are commonly monitored by vibrations, $A E$ has special relevance in low speed components. The low speed part of the wind turbine rotates in a range of 6-20 rpm. With the aim of register the information of at least a entire revolution several seconds must be recorded. This means a lot of memory because the acquisition rate of the $A E$. The device works isolated in the middle of the sea, it is designed to operate independently without access to the wind farm network so the data that is sent for onshore analysis is limited to a few amount of numerical values. The ability to identify errors and defects from parameters extracted form the $A E$ is mandatory.

In order to simplify the computational handling of the data, statistical indicators are employed for the fault detection regarding variations in their levels. This study takes into account the application of this technique for the condition monitoring maintenance of remote machines, trying to make a simple and robust monitoring, without or with the minimum human supervision. Employed with other technologies as vibration, indicators has been 
observed as a realiable and sensitive detection tool also with $A E[26,27]$. There are another strategies with advanced methods in order to increasing the efficiency in operational management [28], but centering the analysis in the indicators avoid complex algorithms and reduces the necessary data amount. The most commonly used indicators in $A E$ and vibration analysis are the root mean square (RMS), kurtosis, event counts and crest factor among others. Several researches have been done with the purpose of validate its employment in $A E$ condition monitoring taking into account the effect of some of the parameters that influence the signal characteristics as speed, load, lubrication and temperature $[7,29,30]$.

The main goal of the present work is focused in the evaluation of the application of acoustic emission in condition monitoring of geared transmissions. Specially at low rotational speed with a simple and robust signal process. The experiment registers the effect produced by an incorrect alignment of a shaft in $A E$ signals, where the rest of the transmission components are in "healthy" condition but affected indirectly by the error. Another important target is the contrast with acceleration measurements during low speed and varying load operation.

In the present study a misalignment error has been introduced in one of the shafts simulating a fault condition. The incorrect meshing of involved gear teeth at low speed could be an advantageous situation for $A E$ versus accelerometry. Some machines are subject to variable load during its operation, specially wind turbines due to the wind fluctuations. Non-stationary load could be a relevant parameter in the monitoring. A time domain analysis trough condition indicators, as it will be done with the developed offshore monitoring device, is performed. The non-stationary conditions

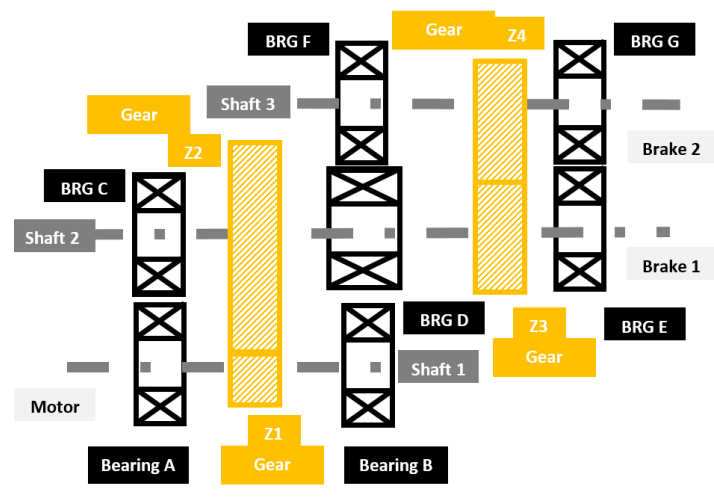

Fig. 1: Gear transmission scheme. over the wind turbine components are working on, simulated in the laboratory test with the varying load, could affect the signals, therefore, the condition indicators. An additional frequency analysis, not available with the device, is carried out.

\section{Experimental procedure}

A set of tests has been performed to determine the effects of shaft misalignment in the acoustic emission signals, its comparison with the vibration analysis and the ability of both methods to recognize the erroneous configuration. It was carried out acquiring signals in a gear transmission, working at different speed and load conditions (stationary and non-stationary), in a reference "healthy" behavior and repeating then with a misaligned shaft.

\subsection{Test bench and sensors location description}

For this experimental test a low speed gear transmission is used. It is compounded by three parallel shafts meshed through two pairs of gears. The system is powered by a $1.1 \mathrm{~kW}$ electric geared motor that provides a range of speeds up to the $60 \mathrm{rpm}$ to the input shaft (shaft 1). The first of those pairs, the gears Z1 in shaft 1 and Z2 in shaft 2, has different number of teeth providing a reduction ratio of one third (20/60). The other set of gears has the same number $(31 / 31)$ so the shaft 2 and the shaft 3 rotate at same speed.

The shafts are mounted on seven supports formed by a casing attached to the bench surface, lifting the three shafts with seven bearings ( $B R G \mathrm{~A}-\mathrm{G}$ in Fig. 1) placed inside. These supports can be moved and fixed again in other position in order to introduce misalignment between shafts. The load is introduced to the transmission via two pneumatic

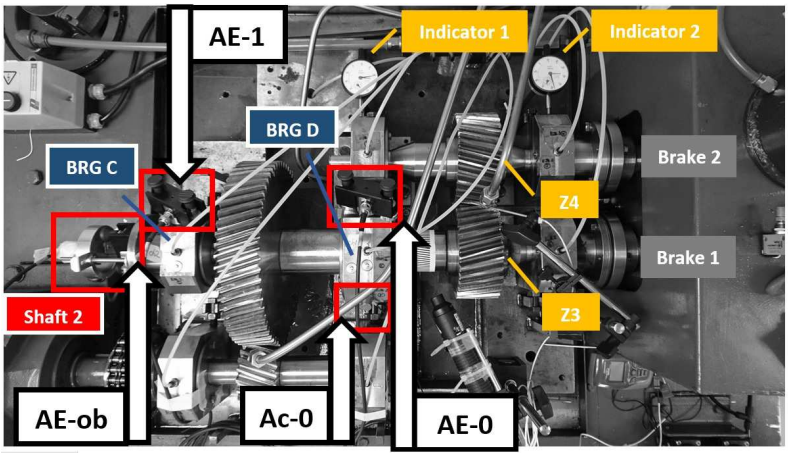

Fig. 2: Sensors emplacement over the transmission. 
brakes placed in shaft 2 and shaft 3 with independent manometers for its separate actuation. All systems (gears and bearings) are lubricated providing a constant flow of oil in the contact areas by direct injection.

The monitoring of the transmission was carried out with three acoustic emission channels, employing $3 A E$ preamplified sensors (VS150-RIC), collected by a commercial 16-bit data acquisition board (AdLink USB-1210). The sensors were placed over a fluid couplant layer, fixed with a magnetic holder and their signals were acquired setting the maximum sample rate of $2 \mathrm{MHz}$ for each channel. This record was performed with a developed software in NI Labview including the simultaneous acquisition of the acceleration signal and a tachometric auxiliary channel, employing another board (NI USB-9162 NI 923). The acceleration was measured with a uni-axial accelerometer (Brüel \& Kjær type 4398).

\begin{tabular}{ccl} 
Point & Channel & Characteristics \\
\hline$B R G B$ & $\begin{array}{c}A E-0 \\
A c-0\end{array}$ & $\begin{array}{l}\text { over the bearing case } \\
\text { placed in parallel to } A E-0\end{array}$ \\
\hline Shaft 2 & $A E-o b$ & on-board at shaft's edge \\
\hline$B R G C$ & $A E-1$ & over the bearing case \\
\hline
\end{tabular}

Table 1: Monitoring points in the transmission.

To obtain further information of the acoustic emission signals produced in the interaction between moving components, the sensors were located in points of different characteristics for a simultaneous acquisition. In addition to these measures, the lubricating oil and ambient temperature were recorded using a datalogger thermometer at all times. Three places were selected for the recording of the acoustic emission and acceleration signals through three $A E$ sensors and one accelerometer.

\section{$B R G D$}

This bearing is located in the center of shaft 2 between the two pair of meshing gears. The acoustic emission channel $A E-O$ and the acceleration channel $A c-0$ were placed over its case in order to compare both technologies. It is different than the rest of the bearings of the bench, it is bigger and it has only one line of rollers. This bigger size of the elements implies more recognizable acoustic emission sources and mayor theoretical frequencies (ball pass over inner race, outer race, etc) than the other models of bearings (smaller) employed in the transmission. In opposition to the behavior of the other bearings, the frequency analysis at this low speed regimen is more clear, where the shaft frequency (and his harmonics) and the bearing frequencies are closer and could be overlapped.

\section{Shaft 2}

Placing a sensor directly on-board in the shaft, the interfaces between itself and the meshing surfaces are avoided, without presence of bearings in the acoustic waves propagation path. The acoustic emission sensor corresponding to the channel $A E$ $o b$ was placed there. There are no interfaces between sensor and source, the gear Z3 and the solid shaft 2 were sturdily fixed. This sensor rotates with the shaft, the signal being extracted through a device that incorporates a slip ring.

$B R G C$

Another acoustic sensor, channel $A E-1$, was placed in this bearing case between the previous two points in order to compare the acoustic emission signals acquired in the main bearing $(B R G D)$.

\subsection{Test description}

The initial aligned condition of the test is set as non-failure or reference condition. The initial position of the shafts is restricted, and the shaft supports location is measured with the indicators. Before carrying out the tests, the bench is operated during enough time in order to warm up the brakes and the lubricating oil temperature becomes steady avoiding non-desired influences in the generated signals.

\begin{tabular}{ll} 
Speed & $($ shaft 2 \& 3) \\
\hline & $15 \mathrm{rpm}$ \\
& $20 \mathrm{rpm}$ \\
& \\
Power & (Through brake) \\
\hline & $750 \mathrm{~W}$ \\
& $1000 \mathrm{~W}$ \\
& $500 \rightarrow 1000 \mathrm{~W}$ \\
& $1000 \rightarrow 500 \mathrm{~W}$ \\
& $750 \rightarrow 1000 \mathrm{~W}$ \\
& $1000 \rightarrow 750 \mathrm{~W}$
\end{tabular}

Table 2: Speed and load conditions of the tests.

Two low speed values are selected to perform the test combined with several load levels (Table 2 ). Several measurements are carried out for 9 seconds for each load and speed condition, varying the load linearly (as much as possible with the manual manometers) in that time interval. All the load is introduced by shaft 3 brake. All the sensors where placed in shaft 2 allowing its rotation without brake interference. 


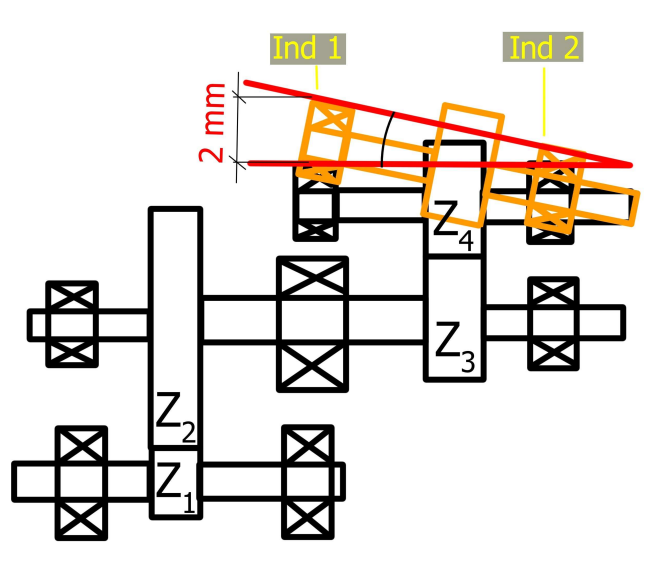

Fig. 3: Misalignment scheme.

Once the normal operation signature is completed, a misalignment error is introduced in the output shaft (shaft 3). Helped with the indicators, the shaft is placed at a new "incorrect" position, imposing an angle between shaft 2 and shaft 3. The support corresponding to indicator 1 , is moved until the gauge has increased by $2 \mathrm{~mm}$ deviation, as is shown in the schematic in Fig. 3, ending with a 1.25 degrees deviation. All tests and calculations are repeated for this configuration.

\section{AE signal processing methods}

The most restrictive $A E$ specifications are the limitations in memory and in the process time. The AE signals are acquired at a sample rate of $2 \mathrm{MHz}$, that means a lot of memory to save and to calculate the monitoring algorithms. The computational cost required to handle multiple big size signal files is important.

Processing methods reducing the data amount are necessary to apply the $A E$ condition monitoring, especially in low speed rotation machines because of the long acquisition time required to record several revolutions. By using this kind of methods, the high frequency AE signals are reduced to a brief description of themselves. When the monitoring tool presents these limitations, the ability to identify the presence of errors and defects trough simplified parameters derived from the AE signal is mandatory.

These limitations are considered in the present work. Therefore, after the record of the measurements, signals are processed with MATLAB calculating the statistical condition indicators to define the signals and applying several methods to perform the analysis.

\section{Condition indicators}

$R M S$ value, kurtosis, crest factor, event counts and 0.99 percentile are assessed in both $A E$ and acceleration signals. A numerical value represents the 9-second-signal facilitating the computational handling and analysis of multiple AE measurements.

The indicators are grouped by speed, load and alignment (correct or misalignment) distinguishing the test conditions. Finally, for each test condition the averages of the indicators are calculated obtaining one representative value for each indicator (Fig. 4). The comparison between alignment and misalignment conditions are evaluated by the percent variations of the averaged indicator value.

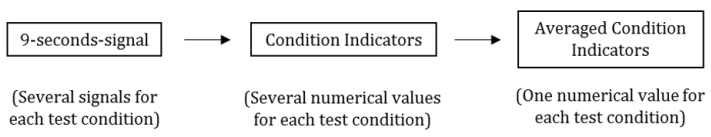

Fig. 4: Time domain indicators method scheme.

Indicator-time evolution signal

The condition indicators set up one characteristic value for the entire 9-second-signal omitting the transitory effects during acquisition time. A method to observe non stationary behaviors in large size AE signals with data reduction without heavy process algorithms is performed.

This method allows to evaluate the time evolution of the signal at low computational cost. A new signal showing the indicator-time evolution is generated by windowing the AE raw signal into many small-time segments in which indicators are calculated (Fig. 5), so as to reflect the transient changes with load variation. Each window data set is averaged with the value of the indicator reducing greatly the amount of data.

Temporal evolution of different signal characteristics is achieved by employing the different statistical indicators, allowing a non-stationary analysis. RMS, or other amplitude related indicators evolution, provides a similar signal than the envelope when the window width tends to zero.

AE envelope

A further analysis of frequencies is carried out to enhance the comparison between acoustic emission 


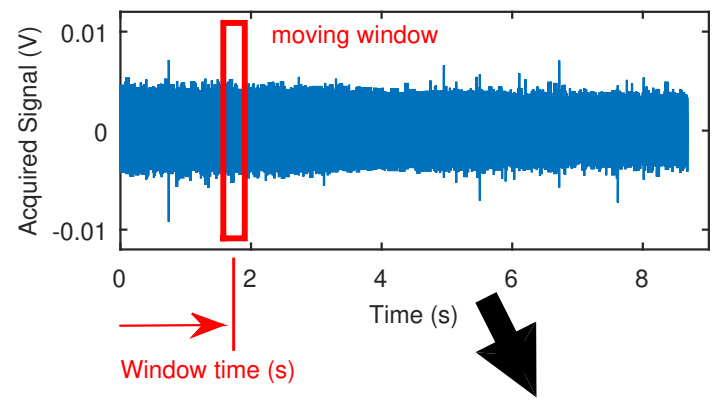

Time evolution:

- RMS

- Kurtosis

- Crest Factor

of moving

window values

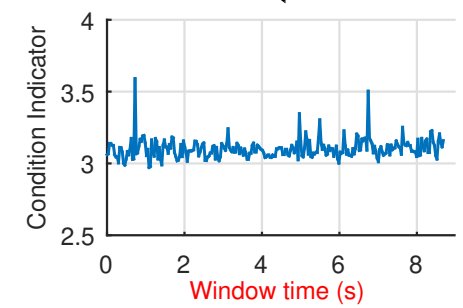

Fig. 5: Scheme of the indicator-time display method employed in the signal analysis.

and acceleration signals. AE is a high frequency signal so additional processing is necessary to evaluate the frequency spectrum at the same range as vibrations. With this aim, the AE signal spectrum is computed based on the signal envelope. The AE envelope is computed by employing band pass filtering and the Hilbert transform.

The identification of a misalignment error and the effect of non-stationary load conditions are assessed by the application of these methods to the signals acquired in the performed tests. Condition indicators offer a clear tool to visualize the defect presence through deviations from the expected reference value when multiple registers have been performed. An in-depth analysis is carried out with the indicator-time evolution and envelope signal.

\section{Results and Discussion}

The main effect of misalignment in the output shaft 3 consists in the modification of the mesh between gears Z3 and Z4. When meshing occurs in the reference condition (aligned) the torque is transferred all over the area of action between the pair of gears. The load distribution along the tooth surface Is modified concentrating the load in one side, an area reduction produces higher pressure in the interaction of both gears. The incorrect contact between teeth is reflected in the increase of peaks amplitude of the on-board sensor $A E-o b$ (Fig. 6).

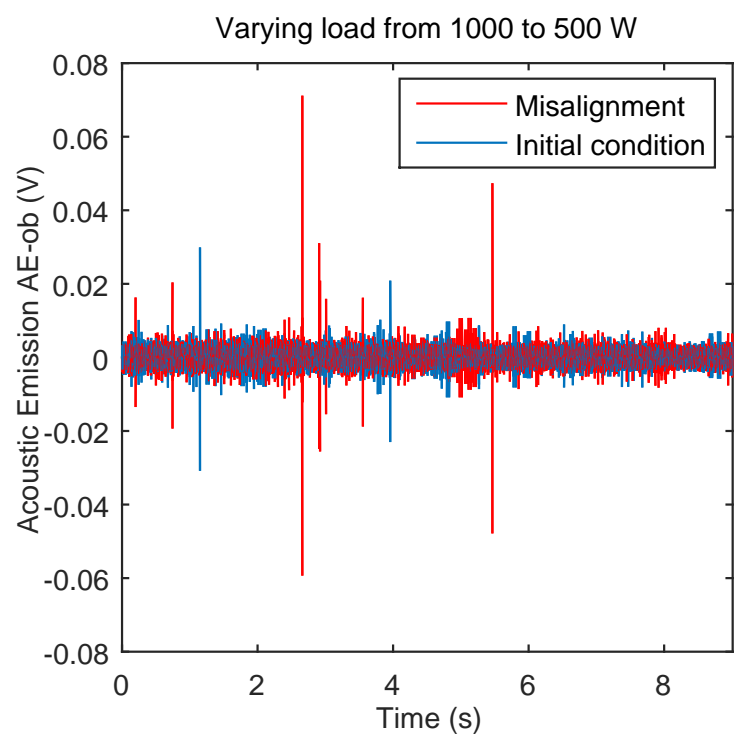

Fig. 6: Effect of misalignment in the on-board $A E$ $o b$ acoustic emission measurements varying load from 1000 to $500 \mathrm{~W}$.

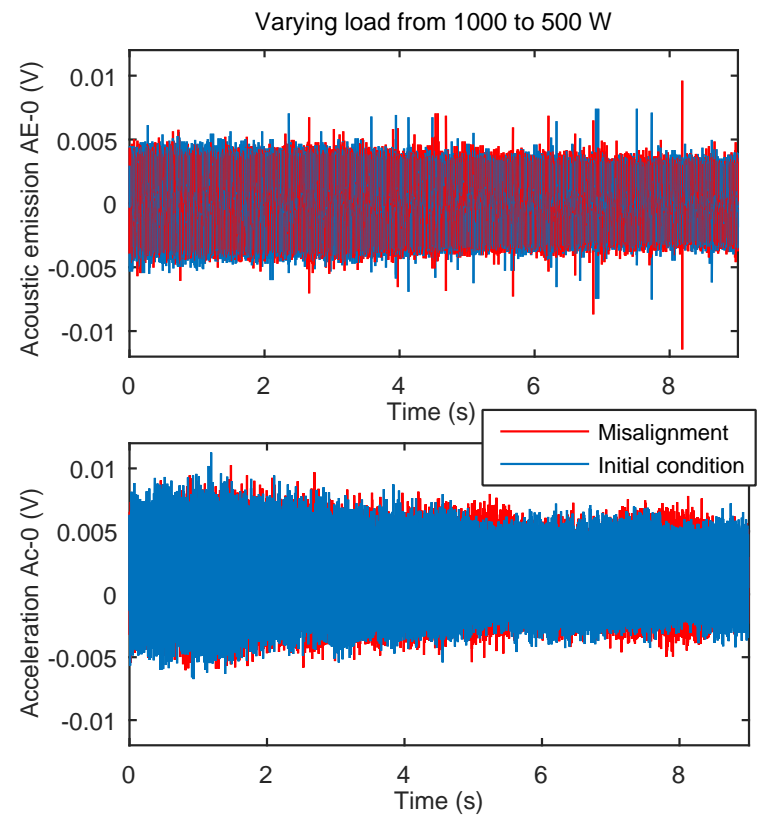

Fig. 7: AE and acceleration 9-second-signals varying load from $1000 \mathrm{~W}$ to $500 \mathrm{~W}$ at $20 \mathrm{rpm}$.

According to the acquired $A E$ signals in the bearing cases, $A E-O$ and $A E-1$, there is no evidence of the teeth contact as it is shown on-board. The higher contact peaks due to the misalignment present in Fig. 6 does not appear in this sensor position. The pressure waves moves through a different path with interfaces and the bearing balls are close to the sensor, generating its own emis- 

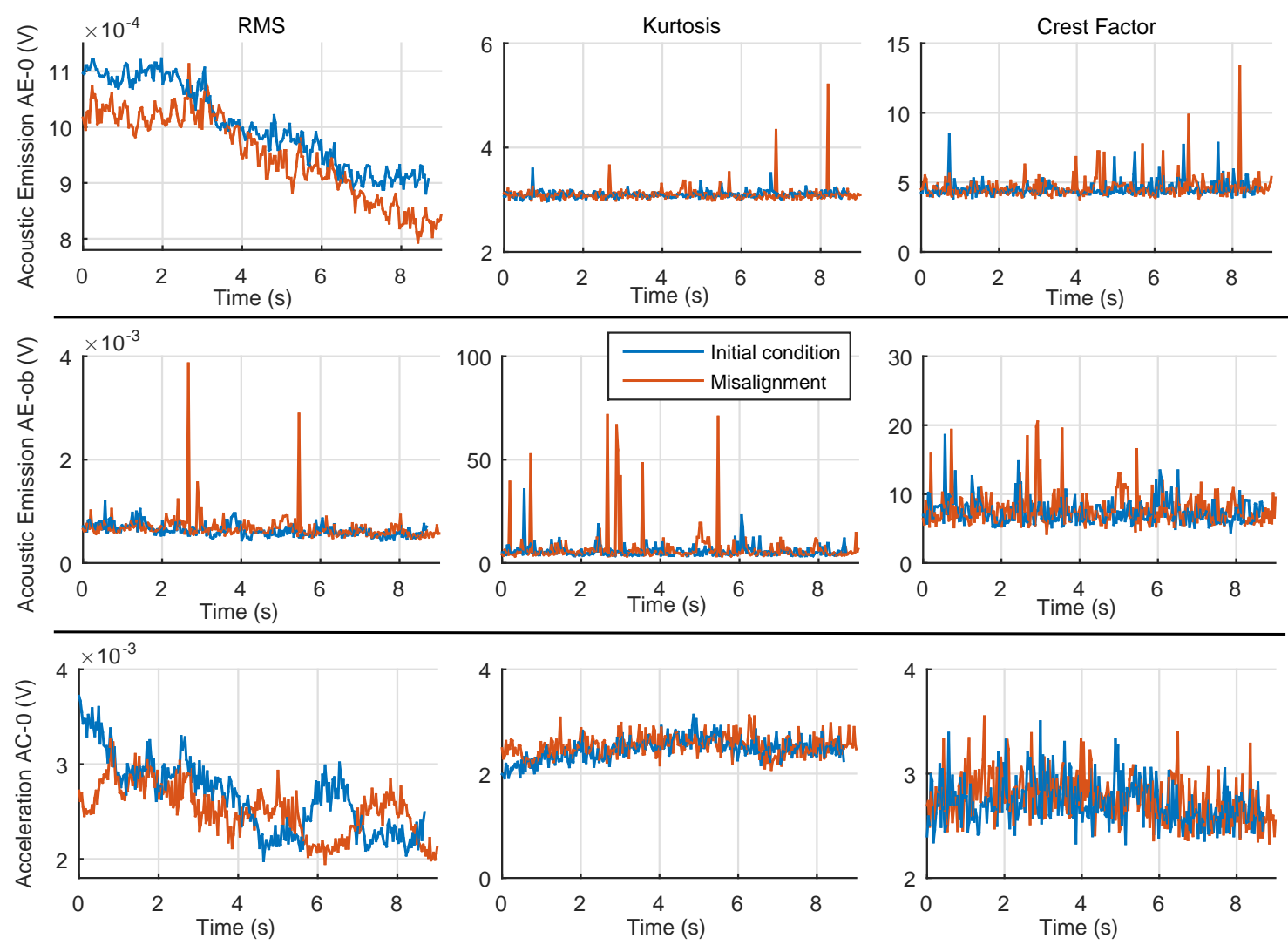

Fig. 8: Signals characterization through indicator-time evolution, varying load from 1000 to $500 \mathrm{~W}$ at 20 rpm.

sions. In these signals there are differences in amplitude when the misalignment is set but the main effect in this way is the load variation. $A E$ signal amplitude, of those channels placed in the bearing cases, follows in linearly trend the variation of the load (Fig. 7). Acceleration signal from Ac-0 placed in the same bearing case as the $A E$ sensor $A E-O$ shows a similar behavior less sharply.

Figure 8 shows the temporal changes of 3 statistic indicators, $R M S$, kurtosis and crest factor, of $A E-0, A E-o b$ and the acceleration channels during the acquisition time of one measure. $R M S$ varies linearly with load in both $A E$ and acceleration signals with and without misalignment regardless of fixed or on-board position.

Misalignment presence is noticed in the $A E-o b$ sensor of shaft 2 where high $R M S$ peaks appears in the same way that the raw $A E$ signal, employing a significantly data reduction. Misalignment causes the appearance of peaks in kurtosis value during measurement time of $A E$ channels, in both fixed and on-board sensors, with a much greater importance in on-board $A E$ - ob sensor for all the slow speed range. On the contrary, it is observed no response in acceleration signal indicators.

\subsection{Monitoring regarding condition indicators}

The condition of the transmission under monitoring is controlled with the values and variations of certain parameters extracted from the measured signals. The presence of the misalignment error is shown through modifications regarding the levels obtained in the "healthy" or reference condition where the shafts are aligned. A very low amount of data is employed, just a few numeric values in front of the signals compounded by data sets with millions of values.

Computing an average of the indicators of all repeated measurements at each same speed and load conditions (Fig. 9 and 10), significant values are obtained in order to compare the misalignment detection through these parameters, with both technologies and different sensor locations. As it has been seen in the time signal characteristics, onboard $(A E-o b)$ measurements are more effective. 

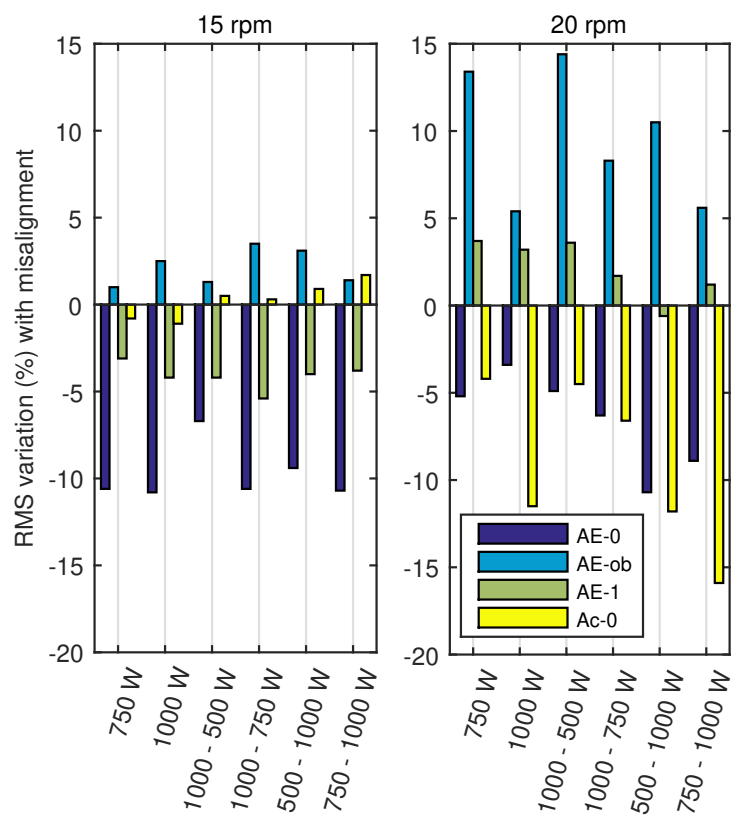

Fig. 9: Percent variation of the average $R M S$ values between reference and misalignment.

The path between the source and this sensor is longer but it does not present discontinuities and moving elements as the fixed sensors over the bearing cases. This clear behavior, explained in the time domain signals, is also reflected in the values of the indicators related with the "peakyness" as the kurtosis (Fig. 10).

The percent variation of the $R M S$ of the onboard channel $A E-o b$ and the acceleration $A c-O$ is more pronounced for higher speeds. If the speed is very low, acceleration indicators are not influenced by fault condition. Acceleration indicators appear to have little relevance for very low speeds. The $R M S$ in the acoustic emission signal of the main bearing $A E-0$ illustrates the presence of defects regardless of the speed (Fig. 9). In the other measured bearing case $(B R G C)$, further and less influenced by the shaft because the position it occupies, the behavior of $A E-O$ channel is reproduced for low velocity, but not when it is operated at a higher speed. This decrease in the $R M S$, in the fixed channel $A E-0$, by introducing the misalignment could be related to the increase in lubrication oil temperature of the bearings which might cause this effect. Between the first measurements and the end of the test, the temperature had a slight increase of $1.5^{\circ} \mathrm{C}$ in the 4 hours, that it has taken to perform the whole test. The low increment and the fact that this does not happen in both bearings studied ( $A E-0$ and $A E-1)$ seems to
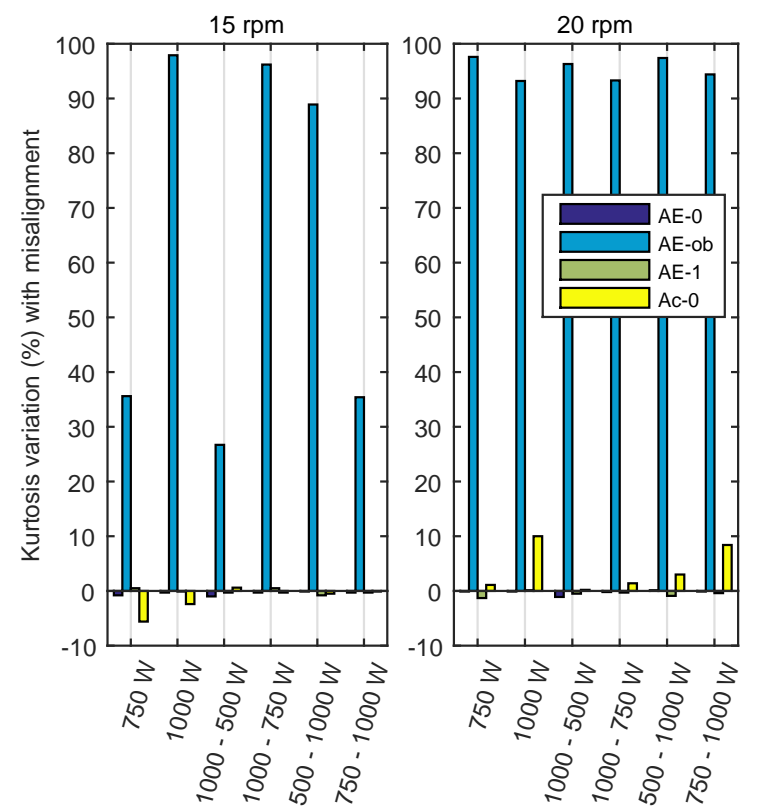

Fig. 10: Percent variation of averaged kurtosis values between reference and misalignment.

indicate that it is an effect in the load sharing due to misalignment, not a response to temperature.

Kurtosis value is triggered in the on-board sensor without affecting the rest of the $A E$ channels or the acceleration (Fig. 10). It is noted that despite the appearance of peaks in the temporal evolution of kurtosis of the $A E-O$ signal on the main bearing (see $A E-0$ temporal evolution of kurtosis with misalignment in Fig. 8), when the average is calculated for the complete-signal-indicators the information is lost and it does not appear in the percent variation. Very few $A E$ kurtosis signal peaks raise above the constant base level of this indicator, so when the kurtosis is evaluated for the entire raw data set it has no impact in the value. The morphology of the acceleration signal, unlike $A E$, does not present this content of "bursts" or peaks. Less variation in the values makes kurtosis not to be a relevant parameter.

4.2 Frequency analysis of the AE envelope and acceleration signals

The acoustic emission signals are acquired at a much higher frequency $(2 \mathrm{MHz})$ than accelerometers (below $100 \mathrm{kHz}$ ), that makes difficult the handling of the data. In spite of the high frequencies of the $A E$ signal, the information of the low frequency events produced in the interaction of the 
bench components can be obtained from its envelope. This method involves a demodulation of the $A E$ signal that obtain frequency ranges similar to acceleration, very useful when the computational cost and data transfer capability are not a limitation.

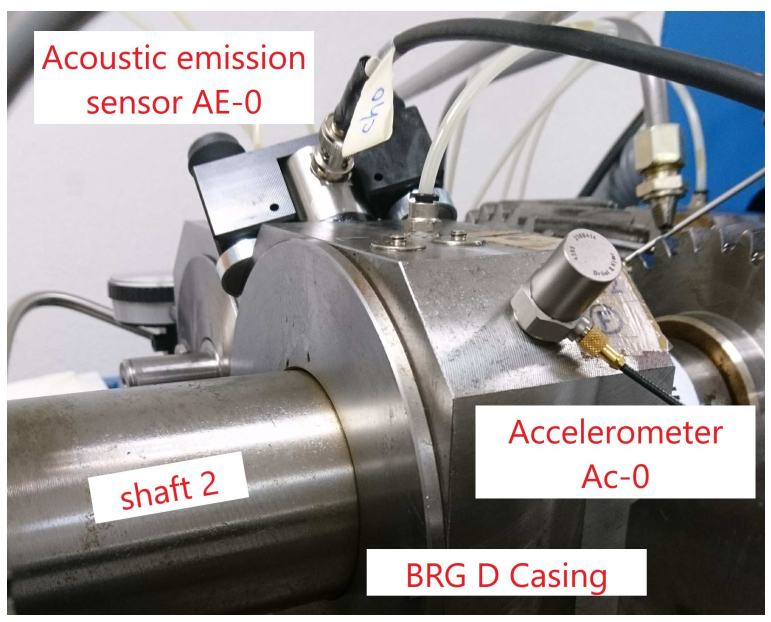

Fig. 11: $A E-0$ and $A c-0$ sensors placed in the same bearing case for the comparison.

The FFT frequency analysis of the envelope $A E$ signals at stationary condition (load and speed) shows the periodicity of the ball pass of the rollers inside the bearing over its outer race. This $B P F O$ frequency, the Ball Pass Frequency of Outer ring, manifests the principal source of the acoustic emission signals in the $A E-O$ channel. The presence of $B P F O$ is clear with the shaft aligned as misaligned in the envelope at stationary conditions (Fig. 12). It shows no response to the misalignment whereas it happens by employing the condition indicators.

In the case of the vibration analysis there is no presence of the $B P F O$ frequency in any configuration, but it reveals the misalignment in the gears Z3 and Z4 mesh frequency (Fig. 12). The 11 $\mathrm{Hz}$ gear mesh frequency is displayed in the spectrum when the transmission is under misalignment. Whenever the machine is working aligned, there is no frequency peak in the acceleration spectrum at this mesh frequency. According to condition indicators calculated from these signals there was not evidence, or they were not clear to define, of the fault presence. The frequency analysis gives a successful detection of the misalignment, an opposite behavior than acoustic emission.

The frequency content of the $\mathrm{AE}$ envelope at the aligned configuration losses the information of the $B P F O$ but it appears in presence of misalign- ment under non-stationary load conditions (Fig. 13). That behavior shows an effect of the misalignment in the acquired $A E$ signals placing the sensors over the bearing cases, registered in the indicators variation. The information does not seem to come directly from the incorrect tooth contacts, there are not frequency content at the meshing frequency. It could be related with the load distribution of shaft and bearing components.

Working under stationary load, the $B P F O$ frequency is present during the $A E$ measurement time for both test condition: aligned and misaligned. The different performances were shown in both test conditions for the $B P F O$ frequency when the conditions are non-stationary. In the misalignment condition, this frequency is also present all across time in spite of the load. It does not show a bet-

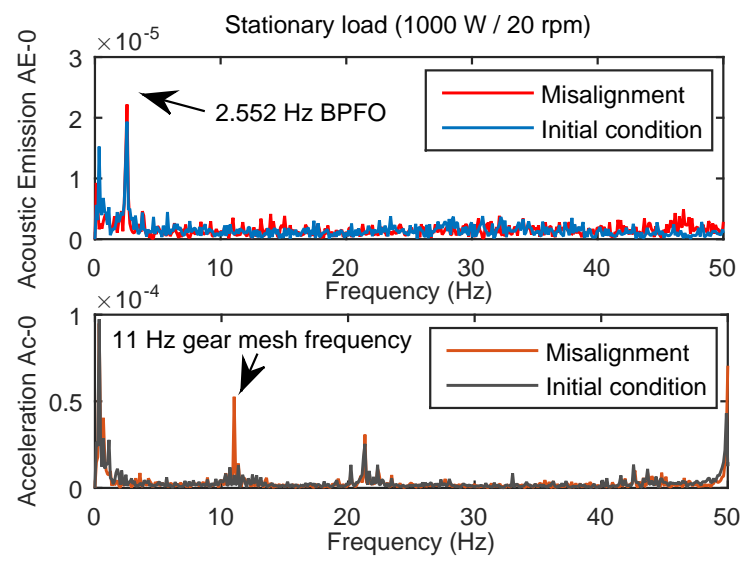

Fig. 12: $A E$ and acceleration frequencies with and without misalignment at the same stationary load condition.
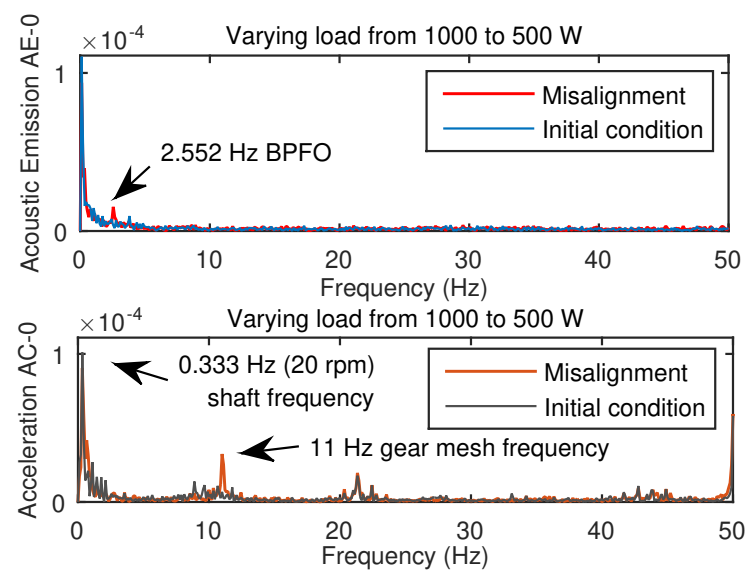

Fig. 13: $A E$ and acceleration frequencies with and without misalignment at the same non-stationary load condition. 

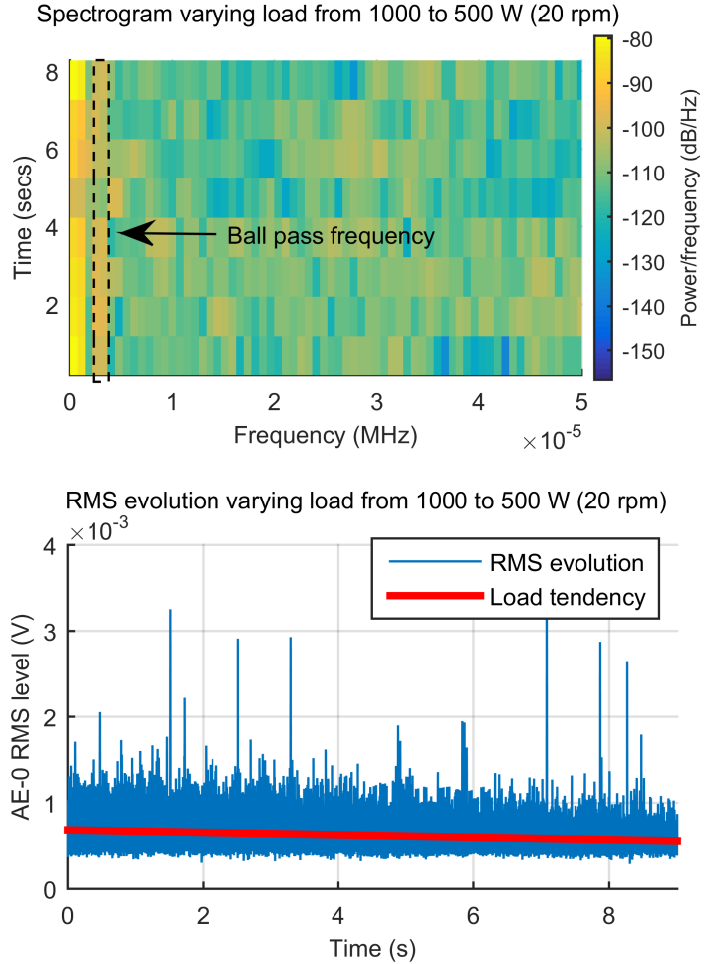

Fig. 14: Spectrogram of $A E$ envelope showing the frequency evolution and (below) RMS variation of the same signal under misalignment and varying load.

ter or worse behavior with high load or low load in the transitory operation (Fig. 14). When the shafts are aligned the $B P F O$ is not noticeable independently of the applied load, using the same varying load levels (Fig. 15). If the frequency evolution of the $A E$ signals is independent regarding the load, it means that the misalignment acts increasing the magnitude of emission source events at the observed frequencies over the perturbations due to the varying load.

The bearing ball pass near the sensor has low $A E$ intensity in the registered signals. It is distinguishable when no perturbations are present at stationary load. When the shafts are aligned the varying load hides the $A E$ ball pass information. It is shown again with the misalignment at varying load. In this case the perturbations does not erase the $B P F O$ because the incorrect gear mesh amplifies the periodic events at this frequency. This could happen, via a different load sharing between shaft and bearing or via a modulation of the noisier signal establishing a path when each ball is closer to the sensor, noticeable with misalignment due higher teeth contact $A E$ bursts.
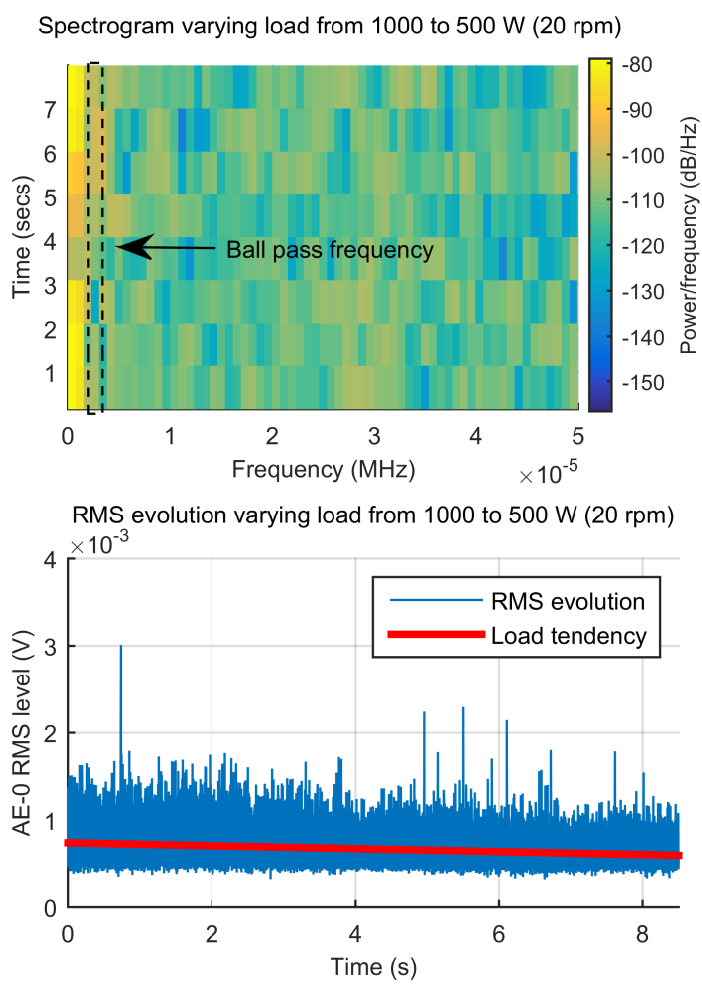

Fig. 15: Spectrogram of $A E$ envelope showing the frequency evolution and (below) RMS variation of the same signal with the shafts aligned and under varying load.

When the shafts work aligned under varying load, the information of ball pass seems to be covered because of the transitory fluctuations. That could mean that non-stationary condition makes the detection of defects unavailable with the $A E$ but the misalignment, generating an incorrect meshing, raises this characteristic frequency content in the $A E$ signals. Non-stationary behaviors is not a limitation of monitoring low speed (and low amplitude signals) misalignment errors with acoustic emission when an abnormal source increases the $A E$ levels due to the defect. The fault configuration was revealed in the time domain condition indicators variation. This frequency analysis correlates its response with mechanical sources.

\section{Conclusions}

In this experimental study signal indicators are used to perform a parameter analysis of the $A E$ signal in order to detect a misalignment error in one of the shafts while the loads are varying. The effect produced by an incorrect alignment of a shaft 
in $A E$ and acceleration signals is evaluated for the monitoring and failure detection of a low speed gear transmission. An analysis in the time domain, signal parameters and frequency domain has been performed in order to discuss its appliance.

Based on the results obtained the location of the sensors proved to be a defining factor. Placing the sensor in direct contact without moving interfaces provides a better $A E$ signal. In this signal the influence of the teeth contacts is present. On the other hand, frequency information of these events is not provided measuring in the bearing case due to the noisy signal. The signal recorded in the bearing case is affected by the rolling elements and interfaces inside the cases.

$A E$ monitoring seems to offer a significant diagnostic advantage at very low speed rates where the indicators of the acceleration signal does not have good sensitivity of the present error. Load variation modifies lineally the amplitude and therefore the RMS values of the signal in the same way with or without misalignment.

An increase in the number of peaks due to the incorrect teeth meshing is produced by the misalignment error in the $A E$ signal therefore kurtosis indicator gives a good response. The detection ability of the indicators is related to the interval of measurement. Sensors on bearing cases detects the fault through other secondary aspects non-directly related with the change in meshing condition.

The frequency analysis of the $A E$ envelope does not show the same information as the acceleration signals. With two sensors placed in the same bearing case, one $A E$ sensor and one accelerometer, the $A E$ collects information of the balls inside the bearing and the accelerometer is not able to do so. On the other hand (in this bench with this misalignment error), the accelerometer can register the presence of misalignment with the appearance of the mesh frequency and the $A E$ sensor shows no frequencies related to the incorrect meshing (sensors placed on bearing casing).

Non-stationary load conditions affect the $A E$ signals. These transitory behaviors are able to provoke the disappearance of the ball pass frequency when the magnitude of the source events is low. In this case, the misalignment error can be distinguished because of its contribution to increase the $A E$ perturbations. However, at stationary load, the difference is not shown just with the frequency information extracted from $A E$ signals. Acceleration frequency analysis performs a clear misalignment monitoring, but regarding its condition indicators a sensitive response is not shown using the accel- eration signals. The information collected by both technologies is not the same, presenting a complementary behavior to the defect detection.

$A E$ condition monitoring of low speed gear transmissions provides better results than vibrations employing the same time domain condition indicators, overtaking the data handling difficulties and avoiding complex signal processing. On the other hand, vibration analysis recognizes clearly the fault through frequency analysis. For $A E$, the interest in frequency analysis is relegated to study the bearing subcomponents. The presence of a non-stationary load during the monitoring has not been seen as a limitation in the application of both technologies.

\section{Acknowledgements}

This work has been supported by project DPI201785390-P funded by the Spanish Ministry of Science and Technology and RM16-XX012 PredictEA project funded by SODERCAN.

\section{References}

1. Beattie AG. Acoustic emission non-destructive testing of structures using source location techniques. Technical report, Sandia National Laboratories, 2013.

2. Kirikera GR, Shinde V, Schulz MJ, Ghoshal A, Sundaresan M, and Allemang R. Damage localisation in composite and metallic structures using a structural neural system and simulated acoustic emissions. $\mathrm{Me}$ chanical Systems and Signal Processing, 21:280-297, 2007 .

3. Mba D and Raj BKN Rao. Development of acoustic emission technology for condition monitoring and diagnosis of rotating machines: Bearings, pumps, gearboxes, engines, and rotating structures. Shock and Vibration Digest, 38(1):3-16, 2006.

4. Ferrando-Chacón JL. Fault Detection in Rotating Machinery Using Acoustic Emission. PhD thesis, Brunel University London, 2015.

5. Coronado D and Fischer K. Condition monitoring of wind turbines: State of the art, user experience and recommendations (project report). Technical report, Fraunhofer IWES, 2015.

6. Mostafapour A. and Davoudi S. Analysis of leakage in high pressure pipe using acoustic emission method. Applied Acoustics, 74(3):335-342, 2013.

7. Tan CK and Mba D. Identification of the acoustic emission source during a comparative study on diagnosis of a spur gearbox. Tribology International, 38(5):469-480, 2005.

8. Sharma RB and Parey A. Modelling of acoustic emission generated in rolling element bearing. Applied Acoustics, 144:96-112, 2019.

9. Rao VV and Ratnam Ch. A comparative experimental study on identification of defect severity in rolling element bearings using acoustic emission and vibration analysis. Tribology in Industry, 37(2):176185, 2015. 
10. Gu D, Kim J, An Y, and Choi B. Low speed rolling bearing fault detection using ae signal. Journal of the Korean Societe of Marine Engineering, 33(5):672$678,2009$.

11. Al-Dossary S and Hamzah RIRand Mba D. Observations of changes in acoustic emission waveform for varying seeded defect sizes in a rolling element bearing. Applied Acoustics, 70(1):58-81, 2009.

12. Qu Y, Van-Hecke B, He D, Yoon J, Bechhoefer E, and Zhu J. A study on comparing acoustic emission and vibration sensors for gearbox fault diagnostics. In MFPT 2014 Conference: Technology Solutions for Affordable Sustainment. Society for Machinery Failure Prevention Technology, 2014.

13. Elforjani MA. Condition Monitoring of Slow Speed Rotating Machinery Using Acoustic Emission Technology. PhD thesis, Cranfield University School Of Engineering, 2010.

14. Molina-Vicuña C. Contributions to the analysis of vibration and acoustic emissions for the condition monitoring of epicyclic gearboxes. $\mathrm{PhD}$ thesis, Aachen: RWTH Aachen University, 2010.

15. Ferrando-Chacon JL, Andicoberry EA, Kappatos V, Papaelias M, Selcuk C, and Gan TH. An experimental study on the applicability of acoustic emission for wind turbine gearbox health diagnosis. Journal of Low Frequency Noise Vibration and Active Control, 35(1):64-76, 2016

16. V. Hariharan and P. S.S. Srinivasan. Vibration analysis of misaligned shaft -ball bearing system. Indian Journal of Science and Technology, 2(9), 2009.

17. Oliver Tonks and Qing Wang. The detection of wind turbine shaft misalignment using temperature monitoring. CIRP Journal of Manufacturing Science and Technology, 17:71-79, 2017.

18. R Errichello and J Muller Geartech. Gearbox reliability collaborative gearbox 1 failure analysis report december 2010 - january 2011. Technical report, NREL, 2012

19. Fernández A, Viadero F, Munoz B, Sancibrian R, and García P. High resolution methods for misalignment detection in low speed gear boxes. IASME Transactions, 1(1):24-29, 2004.

20. Viadero F, Fernández A, Munoz B, Sancibrian R, and García P. A study on comparing acoustic emission and vibration sensors for gearbox fault diagnostics. In Métodos Computacionais em Engenharia. Laboratorio Nacional de Engenharia Civil, 2004.

21. Sako $T$ and Yoshie O. Diagnosis of extremely low-speed rolling-element bearing using ae envelope waveform. IEEJ Transactions on Industry Applications, 132(4):501-509, 2012.

22. Van Hecke B, Yoon J, and He D. Low speed bearing fault diagnosis using acoustic emission sensors. Applied Acoustics, 105:35-44, 2016.

23. Molina-Vicuña $\mathrm{C}$ and Höweler C. A method for reduction of acoustic emission (ae) data with application in machine failure detection and diagnosis. Mechanical Systems and Signal Processing, 97:44-58, 2017.

24. Tan CK, Irving P, and Mba D. A comparative experimental study on the diagnostic and prognostic capabilities of acoustics emission, vibration and spectrometric oil analysis for spur gears. Mechanical Systems and Signal Processing, 21(1):208-233, 2007.

25. Toutountzakis T and Mba D. Observations of acoustic emission activity during gear defect diagnosis. NDT and E International, 36(7):471-477, 2003.
26. Elforjani M, Mba D, Muhammad A, and Sire A. Condition monitoring of worm gears. Applied Acoustics, 73(8):859-863, 2012.

27. Eftekharnejad B and Mba D. Seeded fault detection on helical gears with acoustic emission. Applied Acoustics, 70(4):547-555, 2009.

28. Martinez-Luengo M, Kolios A, and Wang L. Structural health monitoring of offshore wind turbines: A review through the statistical pattern recognition paradigm. Renewable and Sustainable Energy Reviews, 64:91-105, 2016.

29. He Y, Zhang X, and Friswell MI. Defect diagnosis for rolling element bearings using acoustic emission. Journal of Vibration and Acoustics, 131(6):061012106101210, 2009.

30. Molina-Vicuña C. Effects of operating conditions on the Acoustic Emissions (AE) from planetary gearboxes. Applied Acoustics, 77:150-158, 2014. 